\title{
Unified theory for hybrid-photocatalysts: A review on new strategies for synergic, enhanced conversion efficacy and photoredox cycle
}

\author{
Jui-Teng Lin* \\ New Vision Inc., New Taipei City, Taiwan, R.O.C. \\ . *Correspondence: jtlin55@gmail.com
}

\begin{abstract}
. Abstract
This article presents, for the first time, comprehensive model based on the proposed mechanism of Rahal et al [17], Bonardi [20], Tar et al [20], and Mau et al [24], for 3-component systems of G1/Iod/EDB, G1/Iod/amine (in gold chloride), and Iod/Benzoic/Borate (for reduced oxygen inhibition) for the hybri free radical (FRP) and cationic (CP) photopolymerization of interpenetrated polymer network (IPN) systems. Analytic formulas are developed to explore the new features including: (i) conversion efficacy (CE) of FRP is an increasing function of the light intensity (I), the effective absorption coefficient (b), for transient state, whereas, CE at steady-state is independent to the light intensity; (ii) initiator regeneration (RGE) provides a catalytic cycle for improved $C E$ for FRP and CP; (iii) in the IPN system, the synergic effects due to the co-exist of FRP and CP include: (i) CP can increase the medium viscosity limiting the diffusional oxygen replenishment, such that $\mathrm{OIH}$ is reduced; (ii) the cationic monomer also acts as a diluting agent for the radical polymer network, and (iii) the exothermic property of the $C P$ polymerization. We have proposed a scaling law for the transient and steady-state dependence of $\mathrm{CE}$ on the key parameter $\mathrm{P}=\mathrm{bIC}$, given by a an $\mathrm{m}$-order power law of $\mathrm{Pm}$, with $\mathrm{m}=0.5$ to 1.5 , depending on various conditions. The $\mathrm{CE}$ also has an optimal value for maximum CE. The presented comprehensive model (with minimum mathematics) focusing on the enhancement mechanisms/pathways, provides analytic formulas which can be used to analyze reported data, and, more importantly, serves as guidance for exploring new functional materials or new kinetic schemes for improved conversion or procedures for both industrial and medical applications such as additive manufacturing (AM), $3 D$ and $4 D$ bioprinting. Finally, we have proposed new directionslexperiments based on our theoretical predictions.
\end{abstract}

Keywords: polymerization kinetics; conversion efficacy; free radical and cationic radical; photoredox catalyst; $3 D$ bioprinting.

\section{Introduction}

Photopolymerization offers both industrial and medical applications such as dental curing, microlithography, stereolithography, microelectronics, holography [1,2]. Light sources in UV (365 $\mathrm{nm})$ has been conventionally widely, whereas light sources in near-UV (400-410 nm) and visible (430 to $680 \mathrm{~nm}$ ) and near infrared (700 to $1064 \mathrm{~nm}$ ) have been explored more recently in various applications such as additive manufacturing (AM), 3D and 4D bioprinting [3,4]. Both spatial and temporal controlled 3D processes were reported using single and multiple wavelength lights [7-11].

. Organic dyes used as visible light photosensitizers of polymerization have been extensively studied $[12,13]$, in which high extinction coefficients and their long-living excited states, offering high conversion with various additives in the photocurable resins. For cost effective with long-living excited states, copper complexes have been used as a new polymerization for the formation of acetylacetonate radicals by redox reaction with a phosphine and to initiate the free radical polymerization (FRP) of acrylates or the free radical promoted cationic 
polymerization (CP) of epoxides [14-17]. Recently, Coumarin having dual function of photoinitiators in photooxidation or photo-reduction in visible light were reported [18-20]. The efficiency of copper complex (G1) based photoinitiating systems (G1/iodonium salt (Iod)/ $N$-vinylcarbazole (NVK) was investigated by Mokbel et al [16] for FRP of acrylates and CP of epoxides using light source (LEDs at 375, 395, $405 \mathrm{~nm}$ ). Recently, Rahal et al [18,19] also reported the difunction of Coumarin for 3D printing, and modeling was proposed by Chiu et al [20] to analyze their data. Bonardi et al [21] reported high performance near-Infrared photoinitiating systems in the presence of oxygen. Tar et al [22] reported the novel copper photoredox polymerization for in situ synthesis of metal nanoparticles, and was analyzed by modeling of Lin et al [23]. More recently Mau et al [24] reported the panchromatic (using $455 \mathrm{~nm}$ and $530 \mathrm{~nm}$ visible LED) of copper complexes photopolymerization.

. The present comprehensive model (with minimum mathematics) will focus on the enhancement mechanisms/pathways, provides analytic formulas which can be used to analyze reported data, and, more importantly, serves as guidance for exploring new functional materials or new kinetic schemes for improved conversion or procedures in applications such as additive manufacturing (AM), 3D and 4D bioprinting. The present article will skip most of the detailed derivations of the formulas with an emphasis on the synergic and enhancement schemes, whereas more complex kinetics with detailed mathematical approaches, may refer to the modeling published by Lin et al [7-11,17,23]. Furthermore, a greater details on the schemes of various systems can be found in Lin et al [25].

\section{Methods and Modeling Systems}

\subsection{Photochemical Kinetics (multi-components)}

Photopolymerization in general includes free radical-mediated, cationic and anionic catalyzed, and atom transfer radical polymerization. In a radical-mediated photopolymerization, the monomer is converted to polymer after the light irradiation of the photoinitiator (PI) or photosensitizer (PS). The UV (or visible) light produced triplet excited state (T) can couple with: (i) the monomer [M], (ii) the oxygen (if system is in air), or (iii) additives (or co-initiators) producing reactive radicals, which convert the monomer to polymer, The chain growth of a polymer radical with m-links stops as a result of chain termination reactions, Termination of the radicals can occur due to self-recombination, radical-radical coupling, or reacting with the additives. Each radical becomes the center of origin of a polymer chain. Kinetic equations of an m-component radical photopolymerization process may be described as follows.

We will consider a $(\mathrm{n}+1)$-component system, with an initiator [A], triplet excited state $(\mathrm{T}), \mathrm{n}$ additive $B n(n=1,2,3 .$.$) , and one monomer (M)$, the kinetic equations for each of the component concentration are given by

$$
\begin{aligned}
& \frac{d[A]}{d t}=-(b I[A]-R E G) \\
& \frac{d T}{d t}=b I[A]-\left(k^{\prime \prime}+k M+k^{\prime}\left[O_{2}\right]+\sum_{n=1}^{\infty} k_{n} B_{n}\right) T \\
& \frac{d R_{1}}{d t}=k M T+k_{1} B_{1} T+\sum_{n=1}^{\infty} k_{n} B_{n} R_{n}-\sum_{n=1}^{\infty} k_{n} R_{1} R_{n}-K_{1} R_{1} M \\
& \frac{d R_{n+1}}{d t}=k_{n+1} B_{n+1} T+\sum_{n=1}^{\infty} k_{n} B_{n} R_{n+1}-\sum_{n=1}^{\infty} k_{n} R_{n+1} R_{n}-K_{n+1} R_{n+1} M \\
& \frac{d M}{d t}=-k T M-\sum_{n=1}^{\infty} K_{n} R_{n} M
\end{aligned}
$$

Where $b$ is an affective absorption constant proportioanl to the light absorption and excited state quantum yield, I is light intensity, RGE is the initiator-regeneration term. Eq. (2) shows the initiator excited state $(T)$ producing radicals $(\mathrm{Rn})$ by its couplings to the monomer $(\mathrm{M})$, oxygen, and coadditives, $B n$ (with $n=1,2,3 \ldots)$, and having a relaxation rate $k "$. They collectively define the lifetime of $\mathrm{T}$, in which long lifetme leads to larger conversion efficacy. Eq. (3) shows the radical Ri produced from two terms, the type-I, unimolecule cleavage term, kMT; and type-II bimolecular couplings of T and additives $(\mathrm{Bn})$, or enhanced extra radicals $(\mathrm{Rn})$ from its coupling with $\mathrm{Bn}$; the third term is the 
terminations due to radical-radical couplings, including a self-recombination (when $n=1$ ); last term, defines the monomer conversion rate, KR1 due to radical Rn. Eq. (5) defines the monomer conversion total rate function given by the type-I term, kTM, and the sum of all radical-mediated type-II terms, including contributions form additive-enhanced effects via the second term of Eq. (4).

\subsection{Kinetics for a 3-component system $(A / B / N)$}

For comprehensive model, we will consider, as shown by Scheme 1, a 3-component system $(\mathrm{A} / \mathrm{B} / \mathrm{N})$ defined by the ground state of initiator, $[\mathrm{A}]$, which is excited to its first-excited state $\mathrm{PI}^{*}$, and a triplet excited state $T$ which couples with an additive [B] to produce an oxidized-A (or [C]) and a radical $\mathrm{R}$, which interacts with co-additive $\mathrm{N}$ to produce radical $\mathrm{S}^{\prime}$. Further coupling of $\mathrm{S}^{\prime}$ and $[\mathrm{C}]$ produces another radical $S$ and leads to the regeneration of $[\mathrm{A}]$. Monomer $\mathrm{M}^{\prime}$ and $\mathrm{M}$ coupled with radicals $\mathrm{R}$ and $\mathrm{S}^{\prime}$ (for FRP) and S (for $\mathrm{CP}$ ) conversion, respectively.

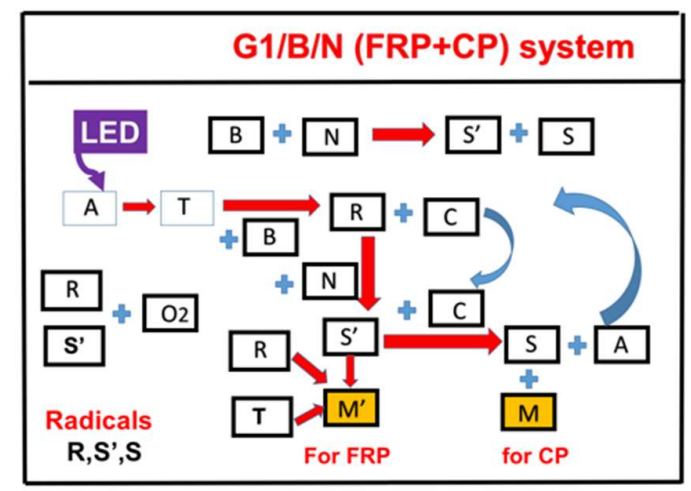

Scheme 1. The scheme chart of a 3-component system, (A/B/N), withe two monomers, $M^{\prime}$ and $M$, for the FRP and CP conversion, respectively, via radicals R, S' and S (see text for details).

Specific measured system related to Scheme 1 was reported by Mokbel et al [16] and a related modeling by Lin et al [17], for a 3-component system of G1/Iod/amine system using a copper complex (G1) and under a $405 \mathrm{~nm}$ LED. More recently, Mau et al [18] proposed the photoredox catalytic cycle for a G2/Iod/EDB system, where G2 is a new copper complex having absorption peaks red-shifted to visible of $455 \mathrm{~nm}$ and $530 \mathrm{~nm}$. The photoredox catalytic cycle for the 3-component system G1/Iod/EDB is shown in Scheme 2 with the associated kinetic reactions shown in Scheme 3: (r1) for the light initiate copper complex (G2) to its excited triplet state $\mathrm{G}^{*}$; (r2) coupling of $\mathrm{G}^{*}$ with Iod salt $\left(\mathrm{Ar}_{2} \mathrm{I}^{+}\right)$to produce oxodized-G2, or G(II), and radical Aro, which, in (r3), couples with EDB to produce radical EDB', which (r4) further couples with $\mathrm{G}(\mathrm{II})$ producing radical $\mathrm{EDB}(+) \mathrm{f}$ and the regeneration of Ge; Also shown is the oxygen inhibition effect $(\mathrm{r} 5)$. The charge transfer between EDB and amine (without the light excitation) producing radicals, $S^{\prime}$ and $S,(r 6)$.

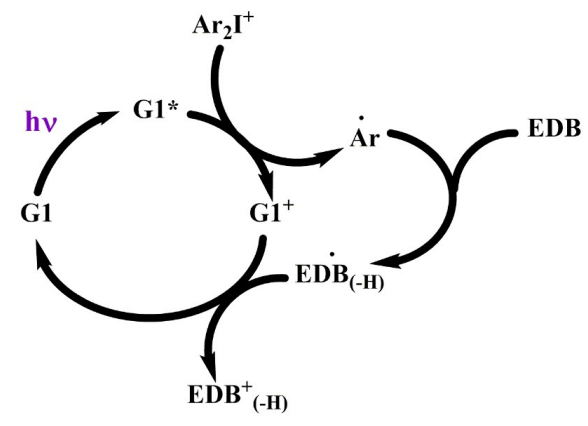


Scheme 2. Photoredox catalytic cycle for the three-component system G1/Iod/EDB [18].

light $+\mathrm{G} 2=\mathrm{G}^{*}$

$\mathrm{G}^{*}+\mathrm{Ar}_{2} \mathrm{l}^{+}=\mathrm{G}(\mathrm{II})+\mathrm{Ar}^{\circ}+\mathrm{Arl}$

$A r^{\circ}+E D B=A r-H+E D B^{\circ}$

$\mathrm{EDB}^{\circ}+\mathrm{G}(\mathrm{II})=\mathrm{EDB}(+)+\mathrm{G} 2$

$\mathrm{Ar}{ }^{\circ}+\mathrm{O}_{2}=\mathrm{Ar}-\mathrm{O}_{2}{ }^{*}$

$\mathrm{Ar}_{2} \mathrm{I}^{+}+\mathrm{EDB}=\mathrm{Ar}^{\circ}+\mathrm{EDB}(+)+\mathrm{Arl}$

Scheme 3. The kinetic reactions for light initiated new copper complex (G2) for both FRP and CP conversation using radicals of $\mathrm{Ar}^{\circ}$ and $\mathrm{EDB}^{\circ}$ (for FRP), and radical $\mathrm{EDB}(+)$ for $\mathrm{CP}$. Also shown are the regeneration of G2 in (r4) and the the oxygen inhibition effect in (r5) and the charge transfer between EDB and amine producing extra radicals, without the light initiation, (r6). See text for more details.

\subsection{Formulas for conversion efficacy (CE)}

The kinetic equations for 3-component system (A/B/N) with 2-monomer M' (for FRP) and M (for $\mathrm{CP})$ are given by the following, under the so-called quasi-steady-state conditions: $\mathrm{dT} / \mathrm{dt}=\mathrm{dR} / \mathrm{dt}=\mathrm{dS} / \mathrm{dt}=\mathrm{dS} / \mathrm{dt}=0$, In addition, the inttiator $[\mathrm{A}]$ rgeneration term (RGE) compensates its light depeltion, i.e, $\mathrm{d}[\mathrm{A}] / \mathrm{dt}=0$, or $[\mathrm{A}]=\mathrm{A}_{0}$, is a constant [ ]. The monomer conversions for FRP and $\mathrm{CP}$ are given by Lin et al $[19,20]$

$\frac{d M \prime}{d t}=-\left(k T+k^{\prime} R+K^{\prime} S^{\prime}\right) M$

$\frac{d M}{d t}=-K S M$

The conversion efficacy (CE) of FRP is defined by $\mathrm{CE}^{\prime}=1-\mathrm{M}^{\prime} / \mathrm{M}^{\prime}$; ; and $\mathrm{CE}$ of of $\mathrm{CP}$ is given by $\mathrm{CE}=1-$ $\mathrm{M} / \mathrm{M}_{0}$, which can be obatin by the time integral of Eq. (1) and (2).

Eq. (1) shows that the CE of FRP has 3 contributions: the type-I from direct coupling of T and M', kTM, and type-II from radical R and S'; whereas the CE of CP is only from one radical of R. Therefore, FRP has high $C E$ than that of $C P$, in general. For exampe, in the system of G2/Iod/EDB [ \}, both radicals $\mathrm{Ar}^{\circ}$ and $\mathrm{EDB}^{\circ}$ ) lead to FRP (in monomer TMPTA), and has a higher conversion efficacy than that of $\mathrm{CP}$ (in monomer EPOX), which is produced by only one radical $\mathrm{EDB}(+)$. As shown by the kinetic equations shon in Scheme 3. Eq. (14) and (15), the rate function of FRP is about twice of CP.

Under the quasi-steady-state, the parameters of Eq. (1) and (2) are given by, Lin et al [ ]: $\mathrm{T}=\mathrm{bIg}[\mathrm{A}], \mathrm{S}^{\prime}=\left[\left(\mathrm{K}_{12}[\mathrm{~B}]+\mathrm{k}_{6} \mathrm{R}\right) \mathrm{N}+\mathrm{k}_{2} \mathrm{~T}[\mathrm{~B}]\right] /\left(\mathrm{K}^{\prime} \mathrm{M}^{\prime}\right) ; \mathrm{S}=\left(\mathrm{K}_{12} \mathrm{~N}+\mathrm{k}_{2} \mathrm{~T}\right)[\mathrm{B}] /(\mathrm{KM}) ;$ and $\mathrm{g}=1 /\left(\mathrm{k}_{5}+\mathrm{k}_{2}[\mathrm{~B}]+\mathrm{kM}^{\prime}\right)$. The oxygen inhibition effect, included in $\mathrm{g}^{\prime}$ and $\mathrm{S}^{\prime}$, reduces the free radicals, $\mathrm{R}$ and $\mathrm{S}^{\prime}$, and hence the lower the conversion of FRP and $\mathrm{CP}$, if system is in air. The radical $\mathrm{R}$ is a complex function given by the solution of Eq. (3) as follows.

$k^{\prime} R^{2}+G R-H=0$

where $\mathrm{G}=\mathrm{k}^{\prime \prime}\left[\mathrm{O}_{2}\right]+\mathrm{k}_{6} \mathrm{~N}+\mathrm{k}_{7} \mathrm{M}^{\prime}, \mathrm{H}=\mathrm{k}_{2}[\mathrm{~B}] \mathrm{T}$; with $\mathrm{T}=\mathrm{bIg}[\mathrm{A}]$. Solving for $\mathrm{R}$, we obtain

$R=\left(\frac{1}{2 \mathrm{k}^{\prime}}\right)\left(-G+\sqrt{G^{2}+4 \mathrm{k}^{\prime} H}\right)$

Analytic formulas of $\mathrm{R}$ is available under two special cases. 
Case (i) for unimolecular termination dominant, or $G>>k^{\prime} H$, we obtain $R=k 2 b I g([A][B] / G)(1-$ $0.5 \mathrm{H} / \mathrm{G})$, which is a linear increasing function of $\mathrm{H} / \mathrm{G}$, or $\mathrm{bIg}[\mathrm{A}][\mathrm{B}] / \mathrm{G}$, for first-order with $0.5 \mathrm{H}<<\mathrm{G}$. Case (ii) for bimolecular termination dominant, with $H \gg \rightarrow G R$, we obtain, $R=\left[H / k^{\prime}\right]^{0.5}$. a nonlinear function of bI, or $\left.\left(\mathrm{k}_{2} \mathrm{bIg}[\mathrm{A}] \mathrm{B}\right] / \mathrm{kk}^{\prime}\right)^{0.5}$.

\section{3.. Results and discussion}

A full numerical simulation is required for the solutions of Eq. (11)-(17), which will be presenetd elasewhere. We will focus on comprehensive analysis for many features and the enhancement effects related to the measured data of Mau et al [18], based on the analytic solutions.

\subsection{Analytic results}

The solutions of the monomer convertion, given by Eq. (1) and (2) depend on $R$ and the approximate form of the g-factor, $g=1 /\left(\mathrm{k}_{5}+\mathrm{k}_{2}[\mathrm{~B}]+\mathrm{k}_{2} \mathrm{~N}+\mathrm{kM}\right)$, which could be approximated as $g=1 /\left(k_{2}[B]\right)$, or $g=1 / k_{5}$. We will focus on the more common case of $g=1 / k_{5}$. For the 2 -component case of A/B (or G1/Iod), with N=0, the solutions of Eq. (1) and (2) give us the CE for FRP and CP as follows.

Case (A) for unimolecular dominant, $R=H / G$, time integral of Eq. (1) gives the CE' (for FRP) =1$\mathrm{M}^{\prime} / \mathrm{M}_{0}^{\prime}$, given by $[9,23]$

$\mathrm{CE}^{\prime}\left(\right.$ for FRP) $=2\left(k^{\prime} / k_{2}\right) B_{0}[1-\exp (-d t)]$

where $d=\left(k_{2} / k_{5}\right) b I A_{0}$. Therefore, $C E^{\prime}=2 k^{\prime} b I t\left(A_{0} B_{0}\right)(1-0.5 d t)$, which is a nonlinear increasing function of light intensity $(\mathrm{I})$, time $(\mathrm{t})$, and the concenration product $\left(\mathrm{A}_{0} \mathrm{~B}_{0}\right)$, in the transient state; In contrast, the steady-state is given by $C E^{\prime}=2\left(k^{\prime} / k_{2}\right) B_{0}$, which is independent to the light intensity (I) or $A_{0}$, in the case of $\mathrm{N}=0$. Moreoverr, the $\mathrm{CE}$ for $\mathrm{CP}$, is given by half of $\mathrm{CE}^{\prime}(\mathrm{FRP})$, that is $\mathrm{CE}$ (for $\left.\mathrm{CP}\right)=0.5 \mathrm{CE}$ (for $\mathrm{FRP}$ ), for the case of $\mathrm{N}=0$.

Case (B) for bimolecular dominant, $R=\left[H / k^{\prime}\right]^{0.5}$, we obtain[ ]

$C E^{\prime}($ for $F R P)=1-\exp \left[-H^{\prime}(t)\right]$

$H^{\prime}(t)=\left(4 k^{\prime} / \sqrt{d B_{0}}\right)[1-\exp (-d t)]$

which has the special feature that higher light intensity has a lower steady state value than that of

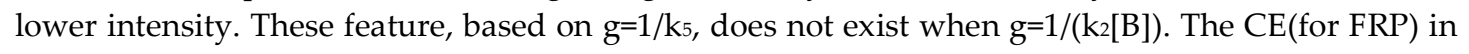
Eq. (6) gives the $\mathrm{CE}($ for $\mathrm{CP})$ with $\mathrm{H}^{\prime}(\mathrm{t})$ reduced to $0.5 \mathrm{H}^{\prime}(\mathrm{t})$.

For 3-component system of $A / B / N$, or G2/Iod/EDB. We need to solve for $[B]$ and $N(t)$. For the strong biomolecular case, we obtain $\mathrm{N}(\mathrm{t})=\mathrm{N}_{0} \exp \left[-\left(\mathrm{Q}^{+} \mathrm{Q}^{\prime}\right)\right], \mathrm{Q}=\left(\mathrm{k}_{6} / \mathrm{k}^{\prime}\right) \mathrm{dB} 0 \mathrm{H}^{\prime}(\mathrm{t}), \mathrm{Q}^{\prime}=\mathrm{K}_{12} \mathrm{~B}_{0} \mathrm{H}^{\prime}(\mathrm{t})$, with $\mathrm{H}^{\prime}(\mathrm{t})=[1-$ $\exp (-\mathrm{dt})] / \mathrm{d}$. Solving for Eq. (16), and for $\mathrm{H}^{\prime}(\mathrm{t})=\mathrm{dt}, \mathrm{N}(\mathrm{t})=\mathrm{Noexp}\left[-\left(\mathrm{Q}^{\prime \prime}\right) \mathrm{t}\right]$, with $\mathrm{Q}^{\prime \prime}=\mathrm{Q}^{+} \mathrm{Q}^{\prime}$, we obtain the $C E$ for FRP as follows [9]

$C E^{\prime}($ for $F R P)=1-\exp [-P(t)]$

$P(t)=H^{\prime}(t)[1+H O R]$

where the high-orer term HOR is a complex function proportional to the time integarl of $\left(\mathrm{K}_{12}[\mathrm{~B}]+\right.$ $\left.\mathrm{k}_{6} \mathrm{R}\right) \mathrm{N}$, which needs numerical integration, having a steady state value proportional to $\left[\mathrm{K}_{12} \mathrm{~B}_{0}+\mathrm{k}_{6}\right.$ $\left.\left(\mathrm{A}_{0} / \mathrm{Ib}\right)^{0.5}\right] \mathrm{N}_{0}$. Tthe CE for $\mathrm{CP}$ is given by Eq (8), but replacing $\mathrm{P}(\mathrm{t})$ to $0.5 \mathrm{P}(\mathrm{t})$.

Based on the CE of eq. (1) to (14), we previously reported, Lin et al [26-28], a general rule having an optimal conditions of the key parameter $\mathrm{P}=\mathrm{bIC}_{0}$ for maximum convesion efficacy (CE). 
Furthermore, the transient and steady-state dependence of $\mathrm{CE}$ on the parameter $\mathrm{P}$, are in general given by a scaling law of $\mathrm{Pm}$, with $\mathrm{m}=0.5$ to 1.5 , depending on various conditions as discussed in the present articles. For examples, $\mathrm{m}=1$ for Eq.(10), and $\mathrm{m}=0.5$ for Eq. (11).

\subsection{The synergic and enhancement effect in IPN system}

\subsubsection{Enhancement strategies}

As reported by van der Laan et al. [4], the effectiveness of a photoinhibitor in a two-color system is strongly monomer-dependent, which also requires: (i) a high conversion of bluephotoinitiation in the absence of the UV-active inhibitor; (ii) a strong chain termination with significant reduction of blue and UV conversion in the presence of UV-active inhibitor and (iii) short induction time or rapid elimination of the inhibitor species in the dark (or absence of UVlight) [22]. Synergic effects have been reported using co-initiator and/or additives and 2- and 3wavelength systems $[8,10]$. Conversion efficiency may be also improved by reduction of the oxygen inhibition effect [23].

\subsubsection{Reduction of oxygen inhibition}

As shown by Scheme 4, the kinetic scheme of a 3-component system, A/B/C in the presence of oxygen, as reported by Bonardi et al [23], and Chiu et al [24]. Under the light exposure, the initiator [A] is exited to its triplet-excited state $\mathrm{T}$, which could react with additive [B] to produce active radical $(\mathrm{R})$ and regenerate $[\mathrm{A}]$. The coupling of radical [R] and oxygen $\left[\mathrm{O}_{2}\right]$ produces a peroxyl radical [ROO], which is too stable for the polymerization to proceed. Therefore, an enhancer [B] is used to create a less stable radical [RO] for extra crosslinks of the monomer, [M]. We note that the co additive [B] plays a dual-function of: (i) regeneration of [A], and (ii) reducing OIH by generating an extra active radical [RO] for improved conversion. Without the dual-function enhancer [B], OIH reduces the radical $[R]$ and lowers the conversion efficacy, in which an induction time is defined for the delayed rising profile of the conversion curve. In the 3-component system of $A / B / C$, notations are associated with Bonardi et al [23] as: [C]= IR-140 borate, [B] = 4-(Diphenylphosphino) benzoic acid (4-dppba), and $[\mathrm{A}]=$ iodonium salt Ar2I+PF6-, with initial concentration of [0.1/2.0/3.0] wt \%, and mixed in a monomer $[\mathrm{M}]=$ methacrylate.

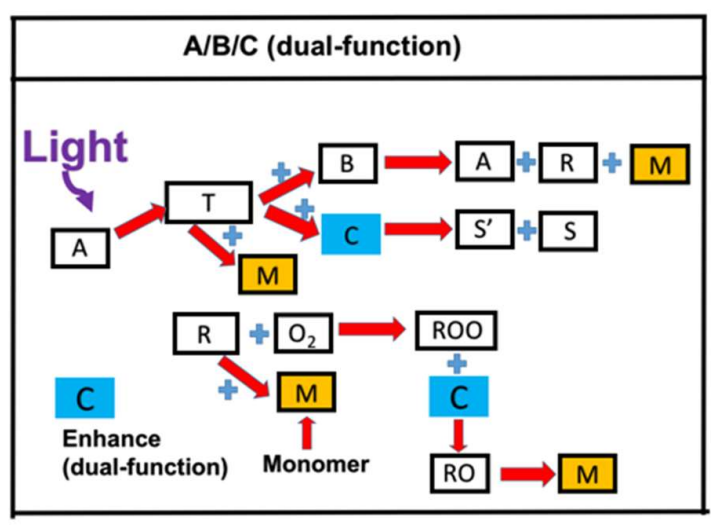

Figure 4. Scheme of $(A / B / C)$ system in which the initiator $[A]$ is excited to its excited triplet state $T$, which could react with $[\mathrm{B}]$ to regenerate $[\mathrm{A}]$, and produce active radical $(\mathrm{R})$. $\mathrm{R}$ could initiate crosslink of the monomer $[\mathrm{M}]$ or react with oxygen producing a radical [ROO], which reacts with $[\mathrm{C}]$ to produce radical [RO] for enhanced conversion [24]. See text for more details.

The monomer conversions for FRP is given, respectively, by [24] 


$$
\frac{\partial \mathrm{M}}{\partial \mathrm{t}}=-\left(\mathrm{bIg}[\mathrm{A}]+\mathrm{k}_{5}[\mathrm{RO}]+\mathrm{k}^{\prime}[\mathrm{R}]\right) \mathrm{M}
$$

where the steady-state radical $[\mathrm{RO}]=\left(\mathrm{k}_{3} / \mathrm{k}_{5}\right)\left([\mathrm{R}]\left[\mathrm{O}_{2}\right] / \mathrm{M}\right)$, with $\mathrm{g}=1 /\left(\mathrm{k}_{5}+\mathrm{k}_{1}[\mathrm{~B}]+\mathrm{k}_{2}[\mathrm{C}]+\mathrm{k}_{7}[\mathrm{M}]\right)$. We note that the radical $[R]$ and conversion is a decreasing function of the oxygen initial concentration, as shown by Eq. (15), whereas conversion is improved by enhancer [C] via its dual function of (i) regenerating $[\mathrm{A}]$, and (ii) generating extra radical [RO], as shown by the second term of Eq. (15), $\mathrm{k}_{5}[\mathrm{RO}]$, which depends on oxygen $\left[\mathrm{O}_{2}\right]$, given by the time integral of $\mathrm{k}_{3}[\mathrm{R}]$, which is given by a solution similar to Eq. (8). The conversion efficacy (CE) of FRP is give by the time integral of Eq. (15), leading to similar formulas as that of Eq. (10) to (14), but having an extra contribution from the enhancer $[C]$, or the $k_{5}[R O]$ term, which is proportional to $k_{3}\left([R]\left[\mathrm{O}_{0}\right] \exp (-Q)\right.$, with $Q(t)$ given by the time integral of $\mathrm{k}_{3}([\mathrm{R}]$.

\subsubsection{Synthesis of nanogold (NG)}

Another example of a 3-component system is in G1/Iod/amine in gold chloride, reported by Tar et al [22] experimentally, and by Lin et al for a modeling [23], as shown in Scheme 4 . The photoinitiating systems are mainly based on G1/TEA/Iodonium salt $(0.05 / 1 \% / 1 \% w / w)$ to the gold chloride $\left(\mathrm{HAuCl}_{4}\right)$ added $4 \mathrm{wt} \%$ in a few drops of DMF, and the PI system was dissolved in EGDA at $93.95 \mathrm{wt} \%$. Scheme 6 shows the proposed kinetics by Tra et al [22]. Using the short hand notations: A= copper complex, $\mathrm{T}=$ excited state, $[\mathrm{HLCuCl}]^{*}, \mathrm{~B}=\mathrm{Iod} ; \mathrm{N}$ is triethylamine $(\mathrm{TEA}), \mathrm{R}=[\mathrm{HLCuCl}]^{+*}, \mathrm{G}=\mathrm{Au}^{+3}, \mathrm{G}^{\prime}=\mathrm{Au}^{+2} \mathrm{Or} \mathrm{Au}^{+1}$. The monomer $(\mathrm{M})$ is EGDA (for FRP conversion). The associated Chart for Scheme 5, is shown in Scheme 6 [23].

\begin{tabular}{|c|c|c|c|}
\hline $\mathrm{Cu}+($ light $)$ & $->$ & $\mathrm{Cu}^{*}$ (excited state) & $(\mathrm{r} 1)$ \\
\hline $\mathrm{Cu}^{*}+\mathrm{Ar}_{2} \mathrm{I}(+)$ & $->$ & $\mathrm{Cu}(+)+\mathrm{Ar}^{\circ}+\mathrm{ArI}$ & $(\mathrm{r} 2)$ \\
\hline $\mathrm{Cu}^{*}+$ amine & $->$ & $\mathrm{Cu}(-)+\operatorname{amine}\left({ }^{\circ}+\right)$ & (r3) \\
\hline amine $\left({ }^{\circ}+\right)$ & $->$ & amine $^{\circ}+\mathrm{H}(+)$ & $(\mathrm{r} 4)$ \\
\hline $\mathrm{Cu}^{*}+\mathrm{Au}(3+)$ & $->$ & $\mathrm{Cu}(+)+\mathrm{Au}(2+)$ & $(\mathrm{r} 5)$ \\
\hline $\mathrm{Cu}^{*}+\mathrm{Au}(2+)$ & $->$ & $\mathrm{Cu}(+)+\mathrm{Au}(1+)$ & (r6) \\
\hline $\mathrm{Cu}^{*}+\mathrm{Au}(1+)$ & $->$ & $\mathrm{Cu}(+)+\mathrm{Au}(0)$ & (r7) \\
\hline $\operatorname{amine}^{\circ}+\operatorname{Au}(3+)$ & $->$ & $\operatorname{amine}(+)+\operatorname{Au}(2+)$ & (r8) \\
\hline $\operatorname{amine}^{\circ}+\operatorname{Au}(2+)$ & $->$ & $\operatorname{amine}(+)+\operatorname{Au}(1+)$ & (r9) \\
\hline $\operatorname{amine}^{\circ}+\operatorname{Au}(1+)$ & $->$ & amine $(+)+\operatorname{Au}(0)$ & (r10) \\
\hline
\end{tabular}

Scheme 5. A proposed kinetics for a system of $\mathrm{Cu} / \mathrm{Iod} / \mathrm{amine}$ in gold chloride $\left(\mathrm{HAuCl}_{4}\right)$ solution, where $\mathrm{G} 1$ (or $\mathrm{Cu}$ ) is for copper complex $(\mathrm{HLCuCl})$, having an excited state $\mathrm{Cu}^{*}$, which couples with iodonium salt, $\mathrm{Ar}_{2} \mathrm{I}(+)$, and amine to produce radicals, $\left(\mathrm{Ar}^{0}\right.$ and amine $\left.{ }^{0}\right)$ which lead to FRP. Both $\mathrm{Cu}^{*}$ and amine $^{0}$ can couple with $\mathrm{Au}(3+)$ to form $\mathrm{Au}(2+)$, then $\mathrm{Au}(1+)$, leading to $\mathrm{Au}(0)$ and the nanogold [22].

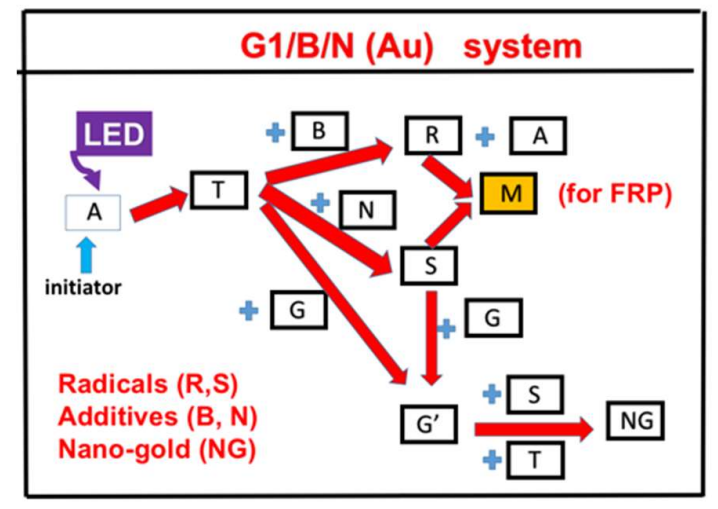


Figure 6. The schematics of a 3-component system, (A/B/N), where $A$ is the ground state of initiator-A, having an excited triplet state $\mathrm{T}$, which interacts with additives $[\mathrm{B}]$ and $[\mathrm{N}\}$ to produce radicals $\mathrm{R}$ and $\mathrm{S}$, which can interact with the monomer (for FRP), or interact with the gold $\left(\mathrm{G}\right.$, or $\left.\mathrm{Au}^{+3}\right)$ to produce exited-gold $\left(\mathrm{G}^{\prime}, \mathrm{or}^{\mathrm{A}} \mathrm{Au}^{+2}\right.$ and $\left.\mathrm{Au}^{+1}\right)$, which then further couples with $\mathrm{T}$ and $\mathrm{S}$ leading to the formation of nanogold $\left(\mathrm{NG}\right.$, or $\left.\mathrm{Au}^{0}\right)$ in the polymer matrix.

The monomer conversions for FRP and generation of nanogold (NG) are given by [23]

$\frac{d M}{d t}=-\left(k T+K R+K^{\prime} S\right) M$

$\frac{d[N G]}{d t}=\left(k_{5} T+k_{6} S\right) G^{\prime}$

Under the quasi-steady-state conditios, $\mathrm{dT} / \mathrm{dt}=\mathrm{dR} / \mathrm{dt}=\mathrm{dS} / \mathrm{dt}=\mathrm{dG} / \mathrm{dt}=0$, which give solutions: $\left.\left.\mathrm{T}=\mathrm{bIg}[\mathrm{A}], \mathrm{R}=\mathrm{k}_{1} \mathrm{bIgg}^{\prime}[\mathrm{A}][\mathrm{B}]\right), \mathrm{S}=\mathrm{k}_{2} \mathrm{bIgg}^{\prime \prime}[\mathrm{A}][\mathrm{N}]\right), \mathrm{G}^{\prime}=\left(\mathrm{k}_{3} \mathrm{~T}+\mathrm{k}_{4} \mathrm{~S}\right) /\left(\mathrm{k}_{5} \mathrm{~T}+\mathrm{k}_{6} \mathrm{~S}\right)$; with $\mathrm{g}=1 /\left(\mathrm{k}_{7}+\mathrm{k}_{1}[\mathrm{~B}]+\mathrm{k}_{2}[\mathrm{~N}]+\right.$ $\left.k_{3} G+k M\right), g^{\prime}=1 /\left(k^{\prime \prime} S+K M\right), g^{\prime \prime}=1 /\left(k_{4} G+k^{\prime \prime} R+K^{\prime} M\right)$. For the case that $g=1 /\left(k_{3} G\right), g^{\prime}=1 /(K M)$, and $\mathrm{g}^{\prime \prime}=1 /(\mathrm{K} ' \mathrm{M})$, we obtain the CE of FRP, which has a similar formula as of Eq. (10) to (14). We note that the FRP is contributed by 3 components: from the coupling of monomer (M) with T, R, and S. The conversion efficacy is proportional to $\mathrm{bI}\left(\mathrm{A}_{0}+k_{1} \mathrm{~B}_{0}+k_{2} \mathrm{~N}_{0}\right) /\left(\mathrm{k}_{3} \mathrm{G}_{0}\right)$.

Production of nanogold, [NG], is given by the solution of Eq. (17), we obtain [23]

$$
[N G](t)=D^{\prime}\left[E(t)+Q^{\prime} E^{\prime}(t)\right]
$$

where $\left.Q^{\prime}=\left(k_{6} k_{2} / k_{3}\right)\left(N_{0} /\left(K^{\prime} M_{0}\right), E(t)=[1-\exp (-d t)] / d\right] ; E^{\prime}(t)=\left[1-\exp \left(-d^{\prime} t\right)\right] / d^{\prime}\right] ; D^{\prime}=b I\left(A_{0} / G_{0}\right) ; d^{\prime}=$ $\mathrm{d}+\left(\mathrm{k}_{1} / \mathrm{k}_{3}\right) \mathrm{D}, \mathrm{D}=\left(\mathrm{k}_{1} / \mathrm{k}_{3}\right) \mathrm{d}\left(\mathrm{A}_{0} / \mathrm{G}_{0}\right)$. We note that $[\mathrm{NG}](\mathrm{t})$ has a transient state value $[N G]=\mathrm{D}^{\prime}\left(1+\mathrm{Q}^{\prime} \mathrm{t}\right)$; and steady-state value, $[N G]=D^{\prime}\left(1 / d+Q^{\prime} / d^{\prime}\right)$, which is independent to the light intensity.

The additive, $[\mathrm{N}]$ (or amine), has dual functions: interacting with $\mathrm{T}$ to produce additional radical (S, or amine ${ }^{0}$ ) which leads to FRP; and coupled with $\mathrm{G}$, or $\mathrm{Au}(3+)$, to form $\mathrm{Au}(2+)$, then $\mathrm{G}^{\prime}$, or $\mathrm{Au}(1+)$, leading to $\mathrm{Au}(0)$ and the nanogold $(\mathrm{NG})$. Both FRP efficacy and the production of NG are proportional to the relative concentration ratios of $\left(\mathrm{A}_{0}+\mathrm{B}_{0}+\mathrm{N}_{0}\right) / \mathrm{G}_{0}$,

\subsubsection{Synergic effects in IPN}

The synergy effects (for improved CE) in an interpenetrated polymer network (IPN) system were discussed by Mau et al [24] for the polymerization of a TMPTA/EPOX blend in the presence of G2/Iod/EDB. Three factors were proposed as follows.

(i) The FRP is at first inhibited by the oxygen in the medium, the OIH effects. However, while the cationic polymerization (CP) starts immediately which increase of the medium viscosity limiting the diffusional oxygen replenishment, such that OIH is reduced; (ii) The cationic monomer also acts as a diluting agent for the radical polymer network allowing to achieve a higher conversion. (iii) the exothermic property of the radical polymerization also tends to boost the cationic polymerization that is quite temperature sensitive. As shown by Eq. (8) and (9), the radical (R) is reduced by the OIH term $\mathrm{k}_{5}\left[\mathrm{O}_{2}\right]$ in $\mathrm{G}$. This OIH effects also supresses the CE of FRP, specialy for the transient profile (till the oxygen is completely depleted). We note that this OIH does not affect the CE of CP, given by the radical $\mathrm{S}$, which is independent to oxygen. This theoretical prediction was justified by the mearsued work of Mokbel et al [19], they reported that the radical for CP is much less sensitive to OIH than that of FRP.

\subsection{General features}


As shown by Eq. (6) and (7) and of Eq. (10) to (15), the following significant features of the system G2/Iod/EDB in monmer blend of TMPTA/EPOX (for FRP and CP, respectively), are summarized.

(a) Under the steady state condition, $\mathrm{bI}[\mathrm{A}]=\mathrm{RGE}$ in Eq. (1), and $\mathrm{d}[\mathrm{A}] / \mathrm{dt}=0$, which leads to a prefect catalytic cycle such that $[\mathrm{A}]$ is a constant., $[\mathrm{A}]=\mathrm{A}$, We note that the REG enhances the conversion of FRP and CP, serving as a catalytic cycle.

(b) Both radicals of $\mathrm{R}$ (or $\mathrm{Ar}^{\circ}$ ) and $\mathrm{S}^{\prime}$ (or EDB') lead to FRP (in monomer TMPTA), and has a higher conversion efficacy than that of $\mathrm{CP}$ (in monomer EPOX), which is produced by only one radical $\mathrm{S}($ or $\mathrm{EDB}(+))$. As shown by Eq. (6) and (7), the rate function of FRP is about twice of $\mathrm{CP}$, when $\mathrm{N}=0$. In the presence of $\mathrm{N}$ (or EDB), efficacy increases due to the coupling terms of $\mathrm{K}_{12} \mathrm{NT}$ in both FRP and CP.

(c) Co-additicve [B] has multiple functions of : (i) regeneration of initiator [A] leading to higher FRP and CP conversion; (ii) producing of radical $\mathrm{S}^{\prime}$ (for FRP), and (iii) producing radical $\mathrm{S}$ (for $\mathrm{CP})$, via the re-coupling with radical $[\mathrm{C}]$, produced by[B].

(d) The oxygen inhibition (OIH) effect (or the term $\mathrm{k}^{\prime \prime}\left[\mathrm{O}_{2}\right]$ ), reduces the free radicals $\mathrm{R}$ and $\mathrm{S}^{\prime}$, and thus the FRP conversion, which is lower in air comparing to in laminate [23]. However, the $\mathrm{OIH}$ effect has much less impact on the $\mathrm{CE}$ of $\mathrm{CP}$, as also demonstrated by Mau et al [24] experimently.

(e) In the IPN system, the synergic effects due to the co-exist of FRP and CP include: (i) CP can increase the medium viscosity limiting the diffusional oxygen replenishment, such that OIH is reduced; (ii) the cationic monomer also acts as a diluting agent for the radical polymer network , and (iii) the exothermic property of the $\mathrm{CP}$ polymerization also tends to boost the cationic polymerization that is quite temperature sensitive. We note that the overall efficacy of both FRP and $\mathrm{CP}$ ate improved via the above described synergic effects, and most importaly, via the catalytic cycle from the regenaration of the initiator [A], the RGE term in Eq. (1) which leads to a constant $[A]$, when RGE $=b I[A]$, such that $d[A] / d t=0$. The present model, for the first time, confirmed mathematically this importnat faeture, which was hypothesized by experimentalists [24].

\section{.3.4 New directions}

Most of the reported experiments are dealing with a fixed light intensity (I) and fixed initiator/additive initial concentrations $\left(\mathrm{C}_{0}\right)$. many of our theoretically predicted features remain to be explored and confirmed experimentally, including the following new directions/topics: (i) optimal conditions [26-28] of the key parameter $\mathrm{P}=\mathrm{blC}_{0}$ for maximum convesion efficacy (CE); (ii) transient and steady-state dependence of $\mathrm{CE}$ on the parameter $\mathrm{P}$, which are in general given by a scaling law of $\mathrm{Pm}$, where $\mathrm{m}=0.5$ to 1.5, depending on various conditins as discuused in the present articles, Eq. (11) to (15); (iii) tehe competing features between FRP and $\mathrm{CP}$, and their dependence on the concentartion ratios, such as $[\mathrm{A}][\mathrm{B}] /[\mathrm{C}]$; (iv) available high efficacy under a minimum dose and/or initiator concentration, for safty issues; (v) enhancements via various strategies of reduction of oxygen inhibition effects, or production of extra radicals; (vi) methods for maximum initiator regeneration (RGE), such that catalytic cyle is available under minimum initiator concentration; (vi) new materials or functional groups for absorption in the visible to near IR range for thick polymers; and (vii) multi-wavelength system for orthogonal type excitation, which is critical in 3D printing, speed and resolutions $[7,8]$.

\section{Conclusion}


10 of 12

We have presented comprehensive model for various systems of G1/ Iod/EDB, and BC/Iod/EPOX systems based on the proposed mechanism of Rahal [17], Bonardi \{20], Tar et al [20], and Mau [24]. Analytic formulas are developed to explore the new features including: (i) efficacy of FRP is an increasing function of the light intensity (I), the effective absorption coefficient (b), and the concentration sum of $\mathrm{A}_{0}\left(\mathrm{k}_{1} \mathrm{~B}_{0}+2 \mathrm{k}_{2} \mathrm{E}_{0}\right)$, for transient state. However, $\mathrm{CE}$ at steady-state is independent to the light intensity; (ii) the efficacy of FRP is an increasing function of the amine (EDB) concentration, in contrast to that of $\mathrm{CP}$ which has an opposite dependence; (iii) initiator regeneration (RGE) provides a catlytic cyle for improved CE for FRP and CP, (iv) In the IPN system, the synergic effects due to the co-exist of FRP and CP include: (i) CP can increase the medium viscosity limiting the diffusional oxygen replenishment, such that $\mathrm{OIH}$ is reduced; (ii) the cationic monomer also acts as a diluting agent for the radical polymer network, and (iii) the exothermic property of the $\mathrm{CP}$ polymerization. Finally, we have proposed new directions, such as new materials or function groups for longer absorption spectrum (in the visible to near IR range) for thick polymers and multiwavelength system based on our theoretcal predictions.

Acknowledgments: JTL thanks the internal grant of New Vision Inc.

Conflicts of Interest: The authors declare no conflict of interest.

\section{References}

1. J.P. Fouassier and J. Lalevée, J. Photoinitiators for Polymer Synthesis-Scope, Reactivity and Efficiency. Wiley-VCH Verlag GmbH \& Co. KGaA: Weinheim, Germany, 2012.

2. Y. Yagci, S. Jockusch and N.J. Turro. Photoinitiated polymerization: Advances, challenges and opportunities. Macromolecules. 2010, 43, 6245-6260.

3. S. C. Ligon, R. Liska, J. Stamp; M. Gurr et al. Polymers for 3D printing and customized additive manufacturing. Chem. Rev. 2017, 117, 10212-10290.

4. B.E. Kelley, I. Bhattacharya, H. Heidari, M. Shusteff et al. Volumetric Additive Manufacturing via Tomographic Reconstruction. Science 2019, 363, 1075-1079.

5. M. P. de Beer, H. L. van der Laan, M.A. Cole et al. Continuous Additive Manufacturing by Volumetric Polymerization Inhibition Patterning. Sci. Adv. 2019, $5,8$.

6. H.L. van der Laan, M.A. Burns, T.F. Scott. Volumetric Photopolymerization Confinement through Dual-Wavelength Photoinitiation and Photoinhibition. ACS Macro Lett. 2019, 8, 899904.

7. J.T. Lin, K.T. Chen, D.C. Cheng, H.W. Liu. Dual-wavelength (UV and Blue) controlled photopolymerization confinement for 3D-printing: modeling and analysis of measurements. Polymers, 2019, 11, 1819.

8. J.T. Lin, K.T. Chen, D.C. Cheng, H.W. Liu. 3-wavelength (UV, blue, red) controlled photopolymerization: improved conversion and confinement in 3D-printing. IEEE Access, 2020, $8,49353-49362$.

9. J.T. Lin, J. Lalevee, D.C. Cheng. Synergetic kinetics of free radical and cationic photopolymerization in three co-initiators and two-monomers system. Polymers (2021, in press).

10. J.T. Lin, K.T. Chen, D.C. Cheng, H.W. Liu. Enhancing blue-light-initiated photopolymerization in a three-component system: kinetic and modeling of conversion strategies. J Polymer Research, 2021, 28:2. 
11. J.T. Lin, K.T. Chen, H.W. Liu, Y.C. Chiu, D.C. Cheng. Enhancing UV photopolymerization by a red-light pre-irradiation: kinetics and modeling strategies for reduced oxygen-inhibition. $\mathrm{J}$ Polymer Science, 2020，58， 683-691，DOI:10.1002/pol.20190201.

12. C. Dietlin, S. Schweizer, P. Xiao, J. Zhang et al. J. Photopolymerization upon LEDs: New Photoinitiating Systems and Strategies. Polym. Chem. 2015, 6, 3895-3912.

13. C. Pigot, G. Noirbent, D. Brunel, F. Dumu. Recent advances on push-pull organic dyes as visible light photoinitiators of polymerization. Eur. Polym. J. 2020, 133, 109797.

14. P. Garra, C. Dietlin, F. Morlet-Savary, F. Dumur et al. Redox two-component initiated free radical and cationic polymerizations: Concepts, reactions and applications. Progress in Polymer Science, 2019, 94, pp.33-56. 10.1016/j.progpolymsci.2019.04.003

15. G. Noirbent, F. Dumur. Recent Advances on Copper Complexes as Visible Light Photoinitiators and (Photo) Redox Initiators of Polymerization. Catalysts, MDPI, 2020, 10, $10.3390 /$ catal10090953.

16. H. Mokbel, D. Anderson, R. Plenderleith, C. Dietlin et al. Simultaneous initiation of radical and cationic polymerization reactions using the "G1" copper complex as photoredox catalyst: Applications of free radical/cationic hybrid photopolymerization in the composites and 3D printingfields. Prog. Org. Coat., 132, 50-61 (2019). DOI: 10.1016/j.porgcoat.2019.02.044

17. J.T. Lin, J. Lalevee, H. Tar, D.C. Cheng. Kinetics analysis of copper complex photoredox catalyst: roles of oxygen, thickness, and optimal concentration for radical/cationic hybrid photopolymerization. Polymers (2021, in press).

18. M. Rahal, H. Mokbel, B. Graff, J. Toufaily et al. Mono vs. Difunctional Coumarin as Photoinitiators in Photocomposite Synthesis and 3D Printing. Catalyst 2020, 10, 1202.

19. Rahal, M.; Mokbel, H.; Graff, B.; Toufaily, J.; Hamieh, T.; Dumur, F.; Lalevée, J. Mono vs. Difunctional Coumarin as Photoinitiators in Photocomposite Synthesis and 3D Printing. Catalyst 2020, 10, 1202.

20. Chiu YC, Cheng DC, Lin JT, Chen KT, Liu HW. Dual-function enhancer for near-infrared photopolymerization: kinetic modeling for improved efficacy by suppressed oxygen inhibition. IEEE Access, 2020, 8, 83465-83471.

21. Bonardi AH, Dumur F, Grant TM, et al. High performance near-Infrared (NIR) photoinitiating systems operating under low light intensity and in the presence of oxygen. Macromolecules, 2018, 51, 1314-1324.

22. Tar, H.; Kashar, T.I.; Kouki, N. et al. Novel Copper Photoredox Catalysts for Polymerization: An In Situ Synthesis of Metal Nanoparticles. Polymers 2020, 12, 2293; doi:10.3390/polym12102293.

23. J.T. Lin, J. Lalevee, H. Tar, D.C. Cheng. Efficacy analysis of in situ synthesis of nanogold via copper/iodonium/amine/gold system under a visible light. Polymers, 2021 (in press).

24. Mau, A.; Mokbel, H.; Dietlin, C.; Graff, B.; et al. Panchromatic Copper Complexes for Visible Light Photopolymerization. Photochem. 2021 (in press). 
25. J.T. Lin, J. Lalevee, H. Tar, D.C. Cheng. Synergic kinetics and strategies for enhanced photopolymerizations for 3D-printing and additive manufacturing - A Critical Review. Polymers, 2021 (in press).

26. Lin, J.T,; Cheng, D.C. Modeling the efficacy profiles of UV-light activated corneal collagen crosslinking. PloS One. 2017;12:e0175002.

27. Lin JT. Photochemical Kinetic modeling for oxygen-enhanced UV-light-activated corneal collagen crosslinking. Ophthalmology Research, 2017;7:1-8.

28. Lin JT. Efficacy S-formula and kinetics of oxygen-mediated (type-II) and non-oxygen-mediated (type-I) corneal cross-linking. Ophthalmology Research. 2018; 8(1): 1-11. 